CONGENITAL HEART DISEASE

\title{
Fetal dextrocardia: diagnosis and outcome in two tertiary centres
}

\author{
A Bernasconi, A Azancot, J M Simpson, A Jones, G K Sharland
}

Heart 2005;91:1590-1594. doi: 10.1136/hrt.2004.048330

See end of article for authors' affiliations

authors affiliations

Correspondence to: Dr Annabelle Azancot, Fetal Cardiology Unit, Hôpital Robert Debré, Paris, France; annabelle. azancot@rdb.ap-hop. paris.fr

Accepted 27 January 2005

\begin{abstract}
Objective: To evaluate the incidence of fetal dextrocardia, associated cardiac and extracardiac malformations, and outcome.

Design: Retrospective echocardiographic study.

Setting: Two tertiary centres for fetal cardiology.

Patients: 81 consecutive fetuses with a fetal dextrocardia presenting at Guy's Hospital, London, between 1983 and 2003 and at Hôpital Robert Debré, Paris, between 1988 and 2003. Fetal dextrocardia was defined as a condition in which the major axis of the heart points to the right.

Results: The incidence was $0.22 \%$. There were 38 fetuses $(47 \%)$ with situs solitus (SS), $24(30 \%)$ with situs ambiguus (SA), and $19(23 \%)$ with situs inversus (SI). Structural cardiac malformations were found in 25 cases $(66 \%)$ of SS, 23 cases $(96 \%)$ of SA, and 12 cases $(63 \%)$ of SI. Extracardiac malformations were identified in 12 cases $(31 \%)$ of SS, in five cases $(21 \%)$ of SA, and in two cases $(10 \%)$ of SI. Of the 81 cases of fetal dextrocardia, there were 27 interrupted pregnancies (15 of $24 \mathrm{SA}, 10$ of $38 \mathrm{SS}$, and 2 of $19 \mathrm{SI})$, six intrauterine deaths ( 3 of 38 SS, 2 of 24 SA, and 1 of 19 SI), and five neonatal deaths ( 3 of 24 SA, 1 of $19 \mathrm{SI}$, and 1 of $38 \mathrm{SS}$ ). There were 43 survivors (24 of $38 \mathrm{SS}, 15$ of $19 \mathrm{SI}$, and 4 of $24 \mathrm{SA}$ ).

Conclusion: The majority of fetuses with dextrocardia referred for fetal echocardiography have associated congenital heart disease. There is a broad spectrum of cardiac malformation and the incidence varies according to the atrial situs. Fetal echocardiography enables detection of complex congenital heart disease so that parents can be appropriately counselled.
\end{abstract}

F etal dextrocardia is a condition in which the major axis of the heart (from the base to the apex along the interventricular septum) points to the right. Dextrocardia should be distinguished from dextroposition, in which the heart is shifted into the right chest as a consequence of pathological states involving the diaphragm, lung, pleura, or other adjoining tissues. ${ }^{1}$ The term dextrocardia describes only the position of the cardiac axis and conveys no information regarding chamber organisation and structural anatomy of the heart. ${ }^{2-7}$ In the postnatal period a broad spectrum of cardiac malformations is observed associated with dextrocardia, and the incidence varies according to the atrial situs. Complex cardiac heart malformations are found more often with situs solitus and situs ambiguus than with situs inversus. ${ }^{367}$ Apart from a few case reports and one recent retrospective study, published data regarding fetal dextrocardia are sparse..$^{-13}$ The purposes of our study was to evaluate the incidence of fetal dextrocardia in our high risk fetal populations and to document the associated cardiac and extracardiac malformations and the fetal outcome.

\section{PATIENTS AND METHODS}

The fetal echocardiographic databases of two fetal cardiology units were retrospectively reviewed (Guy's Hospital, London, from 1983 to 2003 and Hôpital Robert Debré, Paris, from 1988 to 2003) and all patients with fetal dextrocardia were identified. This study group was from a population already preselected for tertiary referral, which may influence the incidence of dextrocardia, as well as associated cardiac malformations and outcome. Fetal dextrocardia was defined as a condition in which the major axis of the heart (base to apex) pointed to the right. The strategy followed for detecting dextrocardia was to determine the left and the right of the fetus based on the position of the spine and head of the fetus (breech, cephalic, transverse) in relation to the maternal abdomen. This technique is learnt from obstetric colleagues in our centres.

From a total of 36765 mothers referred for fetal echocardiography during the study period, 82 cases of dextrocardia were diagnosed. One fetus was excluded because the examination was not technically adequate to define the details of intracardiac anatomy. All fetuses with fetal dextroposition, in which the heart is shifted into the right chest as a consequence of extracardiac abnormality, were excluded from this study. For the 81 fetuses forming the study group, the findings were confirmed by postnatal echocardiography or necropsy, where possible. The gestational age at the time of examination ranged from 17 to 36 weeks, mean (SD) 23.1 (4.7). The referral reason in the majority of cases was a suspicion of cardiac malformation on obstetrical ultrasound $(\mathrm{n}=61)$, followed by extracardiac fetal abnormalities $(\mathrm{n}=9)$, family history $(\mathrm{n}=4)$, fetal dextrocardia $(n=2)$, abdominal situs inversus $(n=2)$, maternal diabetes $(\mathrm{n}=2)$, and nuchal translucency $(\mathrm{n}=1)$. Prenatal records were reviewed in all cases and postnatal clinical records (including necropsy reports and karyotype reports) were reviewed where available. For six babies no follow up information was available. All fetuses had an obstetrical anomaly scan to detect extracardiac malformations. Fetal echocardiograms were recorded with the following equipment: Acuson $128 \mathrm{XP}$, Advanced Technologies Laboratories Ultramark 4 system, Toshiba SSA 270A, and Hewlett Packard 1000, 2000, and 5500 ultrasound systems. Most of the examinations were performed with a 3.5 or $5 \mathrm{MHz}$ transducer. All two dimensional echocardiograms were recorded on videotapes for offline analysis.

Abbreviations: SA, situs ambiguus; $\mathrm{SI}$, situs inversus; SS, situs solitus; VATER, vertebral, anal, tracheal, esophageal, and radial anomalies 


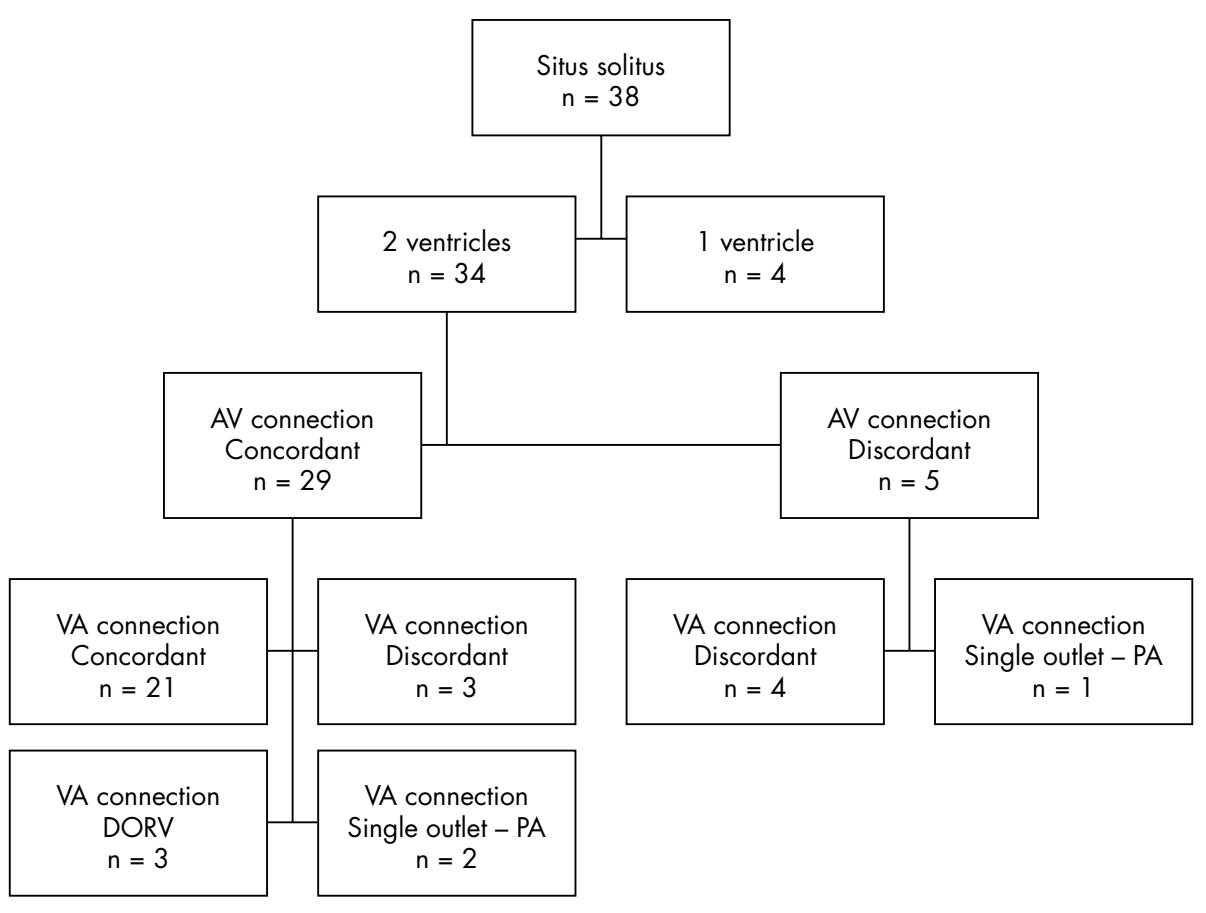

Figure 1 Intracardiac anatomy of fetuses with situs solitus dextrocardia. $\mathrm{AV}$, atrioventricular; DORV, double outlet right ventricle; PA, pulmonary atresia; VA, ventriculoarterial.

The segmental analysis of cardiac anatomy proposed by Tynan et $a l^{14}$ was used for a comprehensive cardiac assessment. Atrial situs was determined by echocardiography according to the location of the inferior vena cava, location of the descending aorta at the level of the diaphragm, and the site of hepatic venous drainage. Situs ambiguus was classified into right and left isomerism. In right isomerism, the inferior vena cava and abdominal aorta lay on the same side of or directly anterior to the spine, with the inferior vena cava running anterior to the aorta. The hepatic veins connected to the inferior vena cava. In left isomerism, the inferior vena cava was interrupted and another venous channel (azygos or hemiazygos) was seen posterior to the aorta. Through this venous channel the abdominal inferior vena cava drained to a superior cava channel. The hepatic veins drained directly to the atria. ${ }^{15}$ We followed the echocardiographic criteria described by Hagler et al ${ }^{16}$ for identification of ventricular morphology.

\section{RESULTS}

From a total of 36765 mothers referred for fetal echocardiography during the study period, 81 cases of fetal dextrocardia were diagnosed, giving an incidence of $0.22 \%$. Fetal dextrocardia was most common with situs solitus in 38 (47\%), followed by situs ambiguus in $24(30 \%)$ and then situs inversus in 19 fetuses $(23 \%)$.

\section{Situs solitus}

There were 38 cases of fetal dextrocardia with situs solitus.

\section{Intracardiac abnormalities}

Thirty four fetuses had two ventricles: 29 had concordant and five had discordant atrioventricular connections (fig 1). The remaining four had univentricular hearts, three with absent right atrioventricular valve and one with double inlet. Of the four fetuses with univentricular hearts, three had univentricular hearts of left ventricular type, all with absent right atrioventricular valve, and one had a univentricular heart of indeterminate type.

Of the 29 fetuses with concordant atrioventricular connections, 21 had concordant arterial connections: 13 had normal hearts and eight had an associated cardiac anomaly. In the remaining eight, three had a discordant ventriculoarterial connection, three had a double outlet right ventricle, and two had a single outlet with pulmonary atresia. In the five fetuses with discordant atrioventricular connections, four had discordant arterial connections and one had a single outlet with pulmonary atresia. Of the three fetuses with a univentricular heart with a main chamber of left ventricular type, two had concordant arterial connections and one had a discordant arterial connection. The other fetus with univentricular heart of indeterminate type had a double outlet main chamber.

\section{Chromosomal anomalies}

Twelve fetuses had documented normal karyotype. In a further 24 fetuses, although fetal karyotype had not been analysed, the karyotype was presumed to be normal, as these had normal phenotype postnatally or at necropsy. One fetus with a ventricular septal defect had trisomy 13, and one fetus with double outlet right ventricle with pulmonary stenosis had balanced 2-9 translocation.

\section{Extracardiac anomalies}

In $31 \%(\mathrm{n}=12)$ of fetuses with dextrocardia with situs solitus an extracardiac anomaly was noted (one VATER syndrome (vertebral, anal, tracheal, esophageal, and radial anomalies), two cleft lip or palate, one Pierre Robin sequence, three polymalformations, one exomphalos, one hydrocephalus, one kidney malformation, one pericardial effusion, and one facial dysmorphia).

\section{Outcome}

Of 38 cases of fetal dextrocardia with situs solitus, $26 \%$ $(\mathrm{n}=10)$ of pregnancies were interrupted, $8 \%(\mathrm{n}=3)$ of pregnancies resulted in spontaneous intrauterine death, and $3 \%(\mathrm{n}=1)$ of babies died after birth. Twenty four survived, representing $86 \%$ of continuing pregnancies.

\section{Situs ambiguus}

Twenty four cases of dextrocardia with situs ambiguus were detected during fetal life. In this group right atrial isomerism 


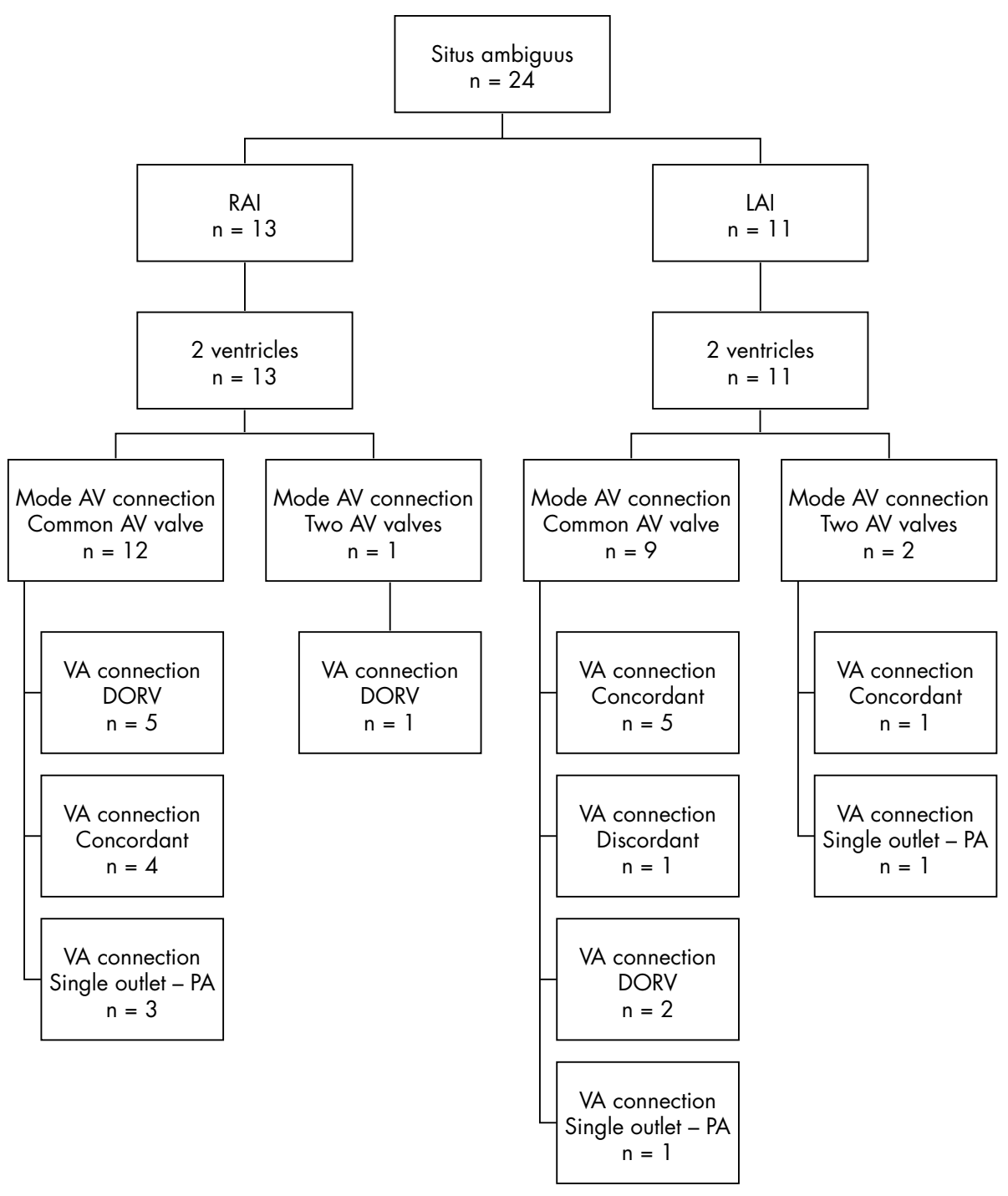

Figure 2 Intracardiac anatomy of fetuses with situs ambiguus dextrocardia. LAI, left atrial isomerism; RAl, right atrial isomerism.

was detected nearly as often as left isomerism (13 and 11 cases, respectively).

\section{Intracardiac abnormalities}

All 13 fetuses with right atrial isomerism had two ventricles, with the morphological right ventricle lying to the right (fig 2). A common atrioventricular valve was found in all but one fetus. Four had a concordant ventriculoarterial connection, six had a double outlet right ventricle, and three had a single outlet with pulmonary atresia. Severe pulmonary stenosis was found in three. Total anomalous pulmonary venous connection was also a common feature and was found in 13 fetuses.

All 11 fetuses with left atrial isomerism also had two ventricles, with the morphological right ventricle to the right. A common atrioventricular valve was found in nine and two fetuses had two patent atrioventricular valves. Six had a concordant and two a discordant ventriculoarterial connection, two had a double outlet right ventricle, and one had a single outlet with pulmonary atresia.

\section{Chromosomal anomalies}

Two fetuses had documented normal karyotype and in 22 cases fetal karyotype had not been analysed.

\section{Extracardiac anomalies}

Three fetuses with left atrial isomerism had gut malrotation and one had polymalformations. One case of oesophageal atresia was found with right atrial isomerism.

\section{Outcome}

In this group $62 \%(n=15)$ of pregnancies were interrupted, $8 \%(\mathrm{n}=2)$ of pregnancies resulted in intrauterine death, $12 \%(n=3)$ of fetuses died after birth, and $17 \%(n=4)$ of the total survived. The survival rate in continuing pregnancy was $44 \%$. The important issue in this type of malformation is the underlying cardiac malformations, although surgical procedures may be more difficult in dextrocardia.

\section{Situs inversus}

There were 19 cases of fetal dextrocardia associated with situs inversus.

\section{Intracardiac abnormalities}

In this group, 18 fetuses had biventricular connections: 17 had concordant and one had discordant atrioventricular connections (fig 3). The remaining fetus had a univentricular heart of the left type with an absent right atrioventricular connection.

Of the 17 fetuses with concordant atrioventricular connections, 12 had concordant ventriculoarterial connections, four 


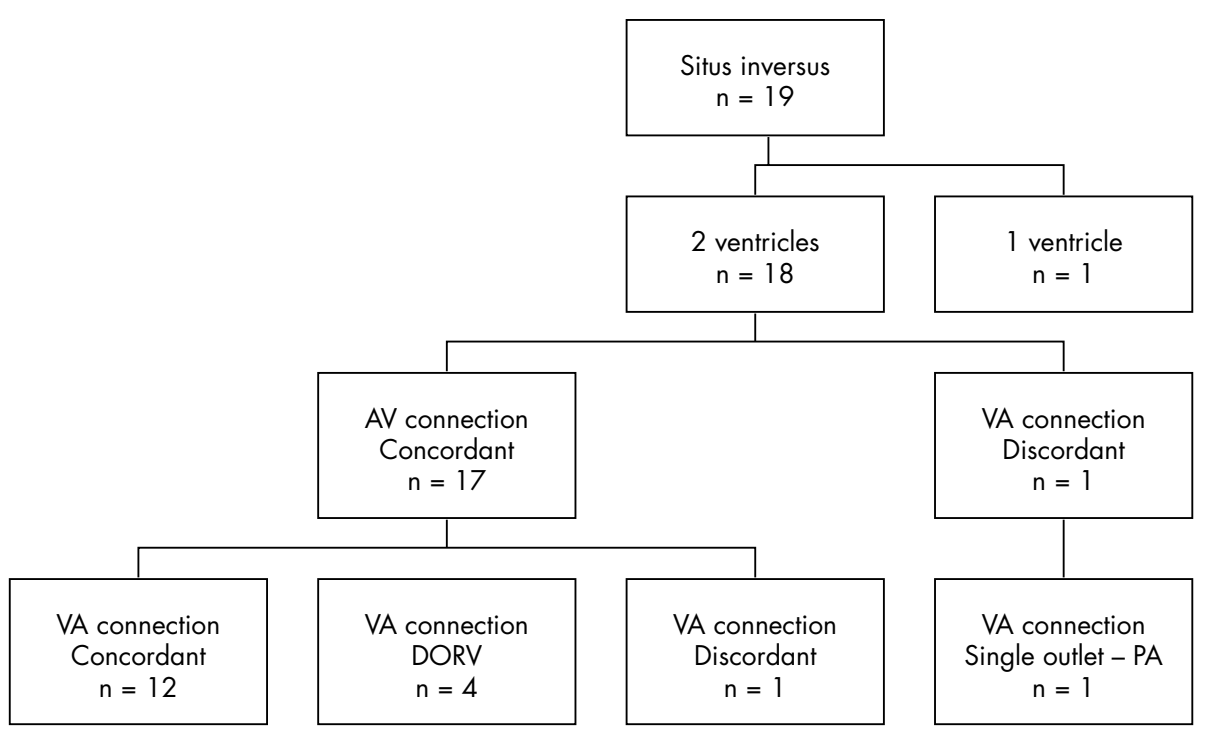

Figure 3 Intracardiac anatomy of fetuses with situs inversus dextrocardia.

had double outlet right ventricle, and one had a discordant arterial connection. Of the 12 fetuses with concordant atrioventricular connections, seven had a normal heart and five had associated cardiac anomalies. The fetus with discordant atrioventricular connection had a single outlet with pulmonary atresia. In the fetus with a univentricular heart the arterial connection was discordant.

\section{Chromosomal anomalies}

Nine fetuses had a documented normal karyotype and in 10 cases fetal karyotype had not been analysed.

\section{Extracardiac anomalies}

Two fetuses with associated ventricular septal defect had extracardiac lesions. One of them had dysplastic kidney and the other the VATER syndrome.

\section{Outcome}

Of the 19 cases of fetal dextrocardia with situs inversus, two pregnancies were interrupted, one resulted in intrauterine death, and one resulted in neonatal death. Fifteen survived, representing $88 \%$ of continuing pregnancies.

\section{DISCUSSION}

\section{Incidence of fetal dextrocardia}

The incidence of fetal dextrocardia in our study population is low $(0.22 \%)$, which is very likely to be related to the referral pattern to our tertiary fetal cardiology units. The referral reason was a suspected cardiac malformation during obstetric ultrasound scanning for most of our patients, with only two patients being referred because of dextrocardia. However, in some of the patients referred for a suspected problem, the dextrocardia had been noted at the time of referral.

The incidence in our series compares with an incidence of $0.84 \%$ in a previous study on fetal dextrocardia (Walmsley et $\left.a l^{13}\right)$. In their study Walmsley et $a l^{13}$ reviewed 5539 fetal echocardiograms and diagnosed 85 cases of dextrocardia. Of these, 46 cases were classified as primary dextrocardia and 39 as secondary dextrocardia due to extra cardiac malformations such as diaphragmatic hernia. In our study, we excluded all cases of secondary dextrocardia or dextroposition related to an extracardiac abnormality. This difference in patient selection may account for some of the differences in the two studies. Dextrocardia is a permanent position of the heart in the right chest, in contrast to dextroposition, which is transitory and regresses when the extracardiac malformation is treated.

\section{Congenital heart malformations associated with dextrocardia}

Both our study and that of Walmsley et $a l^{13}$ show that a wide spectrum of congenital heart malformations, often complex, is associated with fetal dextrocardia. These findings are consistent with postnatal studies. ${ }^{2-7}$ We concur that the term "fetal dextrocardia" indicates cardiac position only and does not give any indications of cardiac structure. As in postnatal studies, we did not find any case of hypoplastic left heart, in contrast to Walmsley et $a{ }^{1,}{ }^{13}$ who found two cases. We did not note an absent left ventricular connection in our study and this finding remains unexplained in the postnatal studies. ${ }^{2}$

\section{The role of situs}

Situs solitus was the most common type in our study $(47 \%)$, which is in contrast to the study of Walmsley et al, ${ }_{13}^{13}$ in which situs solitus was least frequent $(22 \%) .{ }^{13}$ All the fetuses with dextrocardia and situs solitus in the study of Walmsley et $a l^{13}$ had a cardiac malformation compared with $66 \%$ in our study. In postnatal series, the incidence of a normal heart varied between $0-9 \%^{2-7}$ The fetuses with a normal heart in our population were mostly referred because of associated extracardiac malformations and not because there was concern about the fetal heart. This may explain the discrepancies between prenatal and postnatal studies.

In our study $37 \%$ of fetuses with dextrocardia with situs inversus had structurally normal hearts, compared with $89 \%$ in the study of Walmsley et al. ${ }^{13}$ In those with a cardiac malformation a wide spectrum of congenital heart disease was seen, most of which were complex..$^{2-7}$ However, the best survival was observed in this group.

Postnatal series have reported situs ambiguus as the least common type of situs with dextrocardia. However, in prenatal series the incidence is higher, being $29 \%$ in our series and 39\% in the series of Walmsley et al. ${ }^{13}$ The higher incidence in fetal series may be explained by the possibility that many of the fetuses may not survive, either because of interruption of pregnancy or because of death in utero or during the early neonatal period.

\section{Frequency of extracardiac anomalies}

We found extracardiac anomalies in all the three groups of situs. However, only $31 \%(n=25)$ of karyotype results were available. The karyotype was presumed to be normal in liveborn babies with a normal phenotype or in fetuses at necropsy with a normal phenotype. Walmsley et al ${ }^{13}$ 
mentioned extracardiac anomalies only in the situs ambiguus group with one case of trisomy 18. Fetal dextrocardia may be associated with extracardiac anomalies in all types of situs.

\section{Limitations}

Some limitations to our study need to be addressed. The population was preselected by the nature of the referral pattern to our tertiary centres. Thus, the incidence of fetal dextrocardia $(0.22 \%)$ in our population is low and may not be representative of the global incidence. This also applies to the incidence of associated cardiac and extracardiac malformation, which is high in our series. Another potential limitation of our study is the method used to determine the right and left sides of the fetus. This is the first crucial step in obstetric sonography and thus also fetal echocardiography. We have learned from our obstetric colleagues in our specialist centres that the positions of the spine and head of the fetus in relation to the maternal abdomen help work out left and right in the fetus. Cordes et $a l^{17}$ developed a standardised technique to help determine right and left in fetal echocardiography. This method, which also uses the fetal head and spine as markers, is an alternative way to establish the left and right of the fetus. This method has the advantage of being recordable, which is beneficial in retrospective studies.

\section{Conclusion}

A wide spectrum of cardiac disease can be found associated with fetal dextrocardia, depending on the atrial situs. The majority of our fetal population presented because of suspected congenital heart disease during routine obstetric scanning. This is the likely explanation for the low incidence of dextrocardia in our population and accounts for the high incidence of cardiac malformation in our series. The finding of fetal dextrocardia should prompt a comprehensive assessment of fetal cardiac structures by fetal echocardiography. Parental counselling has to take into account whether there is associated congenital heart disease and how severe it is, as these factors will influence prognosis.

\section{Authors' affiliations}

A Bernasconi, A Azancot, Fetal Cardiology Unit, Hôpital Robert Debré, Paris, France

J M Simpson, A Jones, G K Sharland, Fetal Cardiology Unit, Guy's Hospital, London, UK

\section{REFERENCES}

1 Russ PD, Weingardt JP. Cardiac malposition. In: Drose JA, eds. Fetal echocardiography, 1st ed. Philadelphia: WB Saunders, 1998:59-73.

2 Calcaterra G, Anderson RH, Lau KC, et al. Dextrocardia: value of segmental analysis in its categorisation. Br Heart J 1979;42:497-507.

3 Garg N, Agarwal BL, Modi N, et al. Dextrocardia: an analysis of cardiac structures in 125 patients. Int J Cardiol 2003;88:143-55.

4 Huhta JC, Hagler DJ, Seward JB, et al. Two-dimensional echocardiographic assessment of dextrocardia: a segmental approach. Am J Cardiol 1982;50:1351-60.

5 Lev M, Liberthson RR, Ecker FA, et al. Pathologic anatomy of dextrocardia and its clinical implications. Circulation 1968:37:979-99.

6 Squarcia U, Ritter DG, Kincaid OW. Dextrocardia: angiographic study and classification. Am J Cardiol 1974;33:896-903.

7 Stanger P, Rudolph AM, Edwards JE. Cardiac malposition: an overview based on study of 65 necropsy specimens. Circulation 1977;56:159-72.

8 Comstock $\mathrm{CH}$, Smith R, Lee W, et al. Right fetal cardiac axis: clinical significance and associated findings. Obstet Gynecol 1998;91:495-9.

9 De Vore GR, Sarti DA, Siassi B, et al. Prenatal diagnosis of cardiovascular malformations in the fetus with situs inversus viscerum during the second trimester of pregnancy. J Clin Ultrasound 1986;14:454-7.

10 Ohara N, Teramoto K. Situs inversus with dextrocardia diagnosed in the third trimester. J Obstet Gynaecol 2002;22:317-8.

11 Ortiga DJ, Chiba Y, Kanai H, et al. Antenatal diagnosis of mirror-image dextrocardia in association with situs inversus and Turner's mosaicism. J Matern Fetal Med 2001;10:357-9.

12 Pauliks LB, Friedman DM, Flynn PA. Fetal diagnosis of atrioventricular septal defect with dextrocardia in trisomy 18. J Perinat Med 2000;28:412-3.

13 Walmsley R, Hishitani T, Sandor GG, et al. Diagnosis and outcome of dextrocardia diagnosed in the fetus. Am J Cardiol 2004:94:141-3.

14 Tynan MJ, Becker AE, Macartney FJ, et al. Nomenclature and classification of congenital heart disease. Br Heart J 1979;41:544-53.

15 Huhta JC, Smallhorn JF, Macartney FJ. Two dimensional echocardiographic diagnosis of situs. Br Heart J 1982;48:97-108

16 Hagler DJ, Tajik AJ, Seward JB, et al. Atrioventricular and ventriculoarterial discordance (corrected transposition of great arteries) wide-angle two dimensional echocardiographic assessment of ventricular morphology. Mayo Clin Proc 1981;56:591-600

17 Cordes TM, O' Leary PW, Seward JB, et al. Distinguishing right from left: a standardized technique for fetal echocardiography. J Am Soc Echocardiogr $1994 ; 7: 47-53$

\section{FROM BMJ JOURNALS \\ Adverse socioeconomic position across the lifecourse increases coronary heart disease risk cumulatively: findings from the British women's heart and health study}

Please visit the Heart website [www.heartinl. com] for a link to the full text of this article.
Debbie A Lawlor, Shah Ebrahim, George Davey Smith

Objective: To examine the associations of childhood and adult measurements of socioeconomic position with coronary heart disease (CHD) risk.

Methods: Cross sectional and prospective analysis of a cohort of 4286 British women who were aged 60-79 years at baseline. Among these women there were 694 prevalent cases of CHD and 182 new incident cases among 13217 person years of follow up of women who were free of CHD at baseline.

Results: All measurements of socioeconomic position were associated with increased prevalent and incident CHD in simple age adjusted models. There was a cumulative effect, on prevalent and incident CHD, of socioeconomic position across the lifecourse. This effect was not fully explained by adult CHD risk factors. The adjusted odds ratio of prevalent CHD for each additional adverse (out of 10) lifecourse socioeconomic indicator was 1.11 (95\% confidence interval: 1.06, 1.16). The magnitude of the effect of lifecourse socioeconomic position was the same in women who were lifelong non-smokers as in those who had been or were smokers.

Conclusion: Adverse socioeconomic position across the lifecourse increases CHD risk cumulatively and this effect is not fully explained by adult risk factors. Specifically in this cohort of women cigarette smoking does not seem to explain the association between adverse lifecourse socioeconomic position and CHD risk.

A Journal of Epidemiology and Community Health 2005;59:785-793. 